\title{
Inhibition of DACH1 activity by short hairpin RNA represses cell proliferation and tumor invasion in pancreatic cancer
}

\author{
XIAO-NA BU ${ }^{1}$, CHAN QIU $^{1}$, CHUAN WANG $^{2}$ and ZHENG JIANG ${ }^{1}$ \\ ${ }^{1}$ Department of Gastroenterology, The First Affiliated Hospital, Chongqing Medical University, Chongqing 400016; \\ ${ }^{2}$ Department of Gastroenterology, The Third People's Hospital of Chongqing, Chongqing 400014, P.R. China
}

Received January 16, 2016; Accepted February 20, 2016

DOI: 10.3892/or.2016.4843

\begin{abstract}
Cancer of the pancreas is one of the most lethal diseases worldwide. Better understanding of the molecular mechanisms involved in tumorigenesis is of great consequence to elevate the survival rate. Human Dachshund homologue 1 (DACH1) plays a controversial role in human malignancy progression with its expression being altered in a variety of cancers. Nevertheless, its functional roles and molecular mechanisms in pancreatic cancer remain unknown. The expression of DACH1 in pancreatic cancer cell lines and the ductal epithelial cells were evaluated both at mRNA and protein levels. Three pairs of siRNA targeting the DACH1 gene were designed and synthesized, double-stranded short hairpin RNA (shRNA) were annealed and inserted into pGenesil-1 vector, which was confirmed by enzymatic digestion and sequencing analyses. The successfully constructed recombinant plasmids were transfected into Capan-1 cells and our data indicated that knockdown of DACH1 gene expression showed strong correlation with repressing tumorigenesis. The proliferation of Capan-1 cells was significantly repressed as evaluated by CCK- 8 and colony formation assays. Flow cymetry revealed that cell apoptosis was promoted in interference plasmid group compared with control groups $(\mathrm{P}<0.05)$, whereas cell cycle had no significant differences among the groups $(\mathrm{P}>0.05)$. Transwell assay validated the abilities of migration and invasion as being significantly reduced in pshRNA-DACH1 group. Furthermore, our study suggested that DACH1 expression regulates the pancreatic cancer cell apoptosis through interacting with Bcl-2 signaling axis, whereas it controls cell migration and invasion via epithelial-mesenchymal transition (EMT) process.
\end{abstract}

Correspondence to: Professor Zheng Jiang, Department of Gastroenterology, The First Affiliated Hospital, Chongqing Medical University, 1 Yixueyuan Road, Chongqing 400016, P.R. China E-mail: jiangz1753@163.com

Key words: DACH1, pancreatic cancer, invasion, apoptosis, Bcl2, epithelial-mesenchymal transition

\section{Introduction}

Pancreatic cancer is a malignant disease, and the mortality is almost equal to the incident rate due to its aggressive progression and poor prognosis (1). Most pancreatic cancer-related deaths are caused by extremely low diagnostic rate because of its non-distinctive symptoms. Patients with early-stage disease have been reported to have better outcomes, while the majority of patients have no chance of operation once they are diagnosed with terminal-stage, even with metastasis (2). Although, palliative chemotherapy and local chemotherapy are the treatment options for those patients with metastasis, the long-term survival rate is still low. After a long period of searching, more explorations have focused on the promising targeted drugs and the molecular basis of the cancer (3), especially the successful application of FOLFIRINOX protocol (4) and the use of nab-paclitaxel (5), hence, gene therapy is considered as one of the most promising procedures for improving cancer prognoses. Thus, unraveling the molecular mechanisms contributing to pancreatic cancer initiation and progression is urgent.

Recent research has demonstrated the retinal determination gene network (GDRN), a feed-forward transcriptional subcircuit, plays an essential role in regulating cell proliferation and differentiation during embryo development, as well as correlated with cell migration directly (6). Human Dachshund homologue 1 (DACH1) has been implicated as a component of the GDRN, which interacts with other genes of Pax6, Six3, Six 1/2, Eya. Noteworthy, the expression of DACH1 gene is altered in different cancers, it was suppressed in breast, prostate, and gastric cancer (7-9), but overexpressed in ovarian cancer and myeloid leukemia $(10,11)$. However, our previous study detected the expression of DACH1 as significantly upregulated in Capan-1 pancreatic cancer cells compared with the normal counterpart, the immortalized epithelial cell HPDE6-c7, but its role in pancreatic tumor genesis has not yet been fully elucidated.

In our present study, we focused on the effect of DACH1 in tumor cell proliferation and mobility. DACH1 expression was determined both at the mRNA and protein levels via semi-quantitative RT-PCR and western blotting, respectively. Besides, the changes in proliferation and growth of Capan-1 cells after knockdown of DACH1 gene were observed with colony formation and CCK8 assays, while cell cycle and apoptosis were examined with a flow cytometer. Cell migration 
and invasion abilities were then detected by the chamber assay. Furthermore, the signaling proteins involved in the tumor cell apoptosis and invasion were investigated using western blotting.

\section{Materials and methods}

Cell lines culture and transfection. Four pancreatic cancer cell lines (PANC-1, BxPC-3, AsPC-1 and Capan-1), the immortalized pancreatic ductal epithelial cells (HPDE6-c7) and the bacterial liquid pGenesil-1 were obtained from Chongqing Key Laboratory of Molecular Oncology and Epigenetics (The First Affiliated Hospital of Chongqing Medical University, Chongqing, China). The cells were maintained in high-glucose Dulbecco's modified Eagle's medium (DMEM, Hyclone, Logan, UT, USA) supplemented with $10 \%$ fetal bovine serum (FBS, Gibco, Carlsbad, CA, USA) and antibiotics, including $100 \mathrm{U} / \mathrm{ml}$ penicillin and $100 \mathrm{mg} / \mathrm{ml}$ streptomycin (Beyotime Biotechnology, Jiangsu, China). Cells were incubated at $37^{\circ} \mathrm{C}$ in a humidified atmosphere with $5 \% \mathrm{CO}_{2}$ and passaged $1: 3$ once $80 \%$ confluence was reached approximately every 2-3 days. Cells were passaged twice, seeded onto a six-well plate and cultured overnight. Then $250 \mu \mathrm{l}$ serum-free DMEM was separately mixed with $4 \mu \mathrm{g}$ plasmids and $5 \mu \mathrm{l}$ Lipofectamine 2000 (Invitrogen, Carlsbad, CA, USA) for $5 \mathrm{~min}$ at room temperature, subsequently, the diluted plasmid DNA was combined gently with diluted transfected agent Lipo for further $30 \mathrm{~min}$. Then the mixtures without serum were added in each well for cell starvation at $37^{\circ}$ with $5 \% \mathrm{CO}_{2}$ incubator, after $4-6 \mathrm{~h}$ starvation, the medium was changed with the complete medium containing $10 \%$ FBS.

RNA isolation and semi-quantitative RT-PCR analysis. Total RNA was separately isolated from cell lines by using TRIzol reagent (Takara, Dalian, China), reverse transcribed using Go Script Reverse Transcription System (Promega, Madison, WI, USA), and then semi-quantitative RT-PCR was performed with Go Taq ${ }^{\circledR}$ Flexi DNA polymerase (Promega). The temperature profile used for PCR was $95^{\circ} \mathrm{C}$ initial denaturation for $2 \mathrm{~min}$, followed by 36 cycles of $95^{\circ} \mathrm{C}$ denaturation for $30 \mathrm{sec}, 55^{\circ} \mathrm{C}$ primer annealing for $30 \mathrm{sec}$ and $72^{\circ} \mathrm{C}$ extension for $30 \mathrm{sec}$, ultimately with a 3 min extension step at $72^{\circ} \mathrm{C}$. $\beta$-actin was amplified as a positive control with 23 cycles as well. The amplification products were electrophoresed into a $2 \%$ agarose gel (Biowest, Barcelona, Spain), then images were captured by Bio-Rad gel imaging instrument and analyzed with Quantity One software. The primer sets for DACH1 gene were: forward, 5'-TTGCATACGGTCTACACCAAG-3'; reverse, 5'-TGAGAC CAGGGACAGAATGC-3'. $\beta$-actin primers: forward, 5'-CTC CATCCTGGCCTCGCTGT-3'; reverse, 5'-GCTGTCACCTT CACCGTTCC-3'.

Design and synthesis of shRNA. The Homo sapien DACH1 gene sequence (GenBank Accession no. NM_080759.5) was identified in the NCBI gene bank. According to the principles of siRNA design, three small interfering RNA (siRNA) targeting human DACH1 (site at 1325th, 1543th and 1744th base) were designed by primer design software Primer Premier 5 and Oligo 6, which has no sequences homologous with any other gene. Moreover, a 21-base non-specific sequence was designed as a negative control. Then, the short hairpin struc- ture was constructed with a stem-loop TTCG, connecting the DACH1-target siRNA sequence and its reverse complementary sequence. By BLAST with the sequences in the international nucleotide database, the specificities of the three DACH1-target shRNAs were confirmed. These four oligonucleotides were synthesized by Invitrogen (Shanghai, China).

Construction of recombinant plasmid. The four groups of oligonucleotides were separately dissolved in distilled water, making the final concentration at $50 \mu \mathrm{mol} / 1$, and were then mixed with $5 \mathrm{X}$ annealing buffer for DNA oligos according to the manual (Nobleryder, Beijing, China). The mixture was incubated in $95^{\circ} \mathrm{C}$ for $2 \mathrm{~min}$, and then dropped to $4^{\circ} \mathrm{C}$ by gradient cooling every $90 \mathrm{sec}$ in the polymerase chain reaction (PCR) machine. The empty vector pGenesil-1 was extracted from bacterial liquid by E.Z.N.A. Plasmid Mini kit I (Omega Bio-Tek, Norcross, GA, USA) and double digested with BamHI and HindIII enzyme (Takara) in a $37^{\circ} \mathrm{C}$ water bath for 6 h. Afterward, E.Z.N.A Gel Extraction kit (Omega Bio-Tek) was used to recycle the restriction fragment after endonuclease reaction. Subsequently, T4 DNA ligase (Takara) was chosen to connect the shRNA with the purified pGenesil-1 plasmid fragment at $16^{\circ} \mathrm{C}$ in the metal bath overnight. The ligation products were transformed into the competent Escherichia coli cells DH5 $\alpha$ (Tiangen, Beijing, China). Recombinant plasmids were extracted by E.Z.N.A. Plasmid Mini kit I for analysis of enzyme identification and sequencing by Sangon Biotech Co. (Shanghai, China). The correct plasmids were named as pshRNA1-DACH1, pshRNA2-DACH1, pshRNA3-DACH1, pGenesil-1-shRNA-negative control (pshRNA-NC).

Recombinant plasmid transfections and identification of the most effective inhibitory rate group. Endotoxin-free plasmids were extracted using the E.Z.N.A. Endo-Free Plasmid Mini kit I (Omega Bio-Tek). Pancreatic cancer cell line Capan-1, which expresses high level of endogenous DACH1, was transfected with the recombinant plasmids via Lipofectamine 2000 according to the protocol mentioned above. Cells were divided into six groups: pshRNA1/2/3DACH1, pshRNA-NC, pGenesil-1 and untreated cell. Forty-eight hours after transfection, the expression of DACH1 was tested by semi-quantitative RT-PCR and western blotting. The band intensity values were then analyzed with Quantity One software to choose the most effective interference plasmid. Besides, the expression of GFP was observed under an inverted fluorescence microscope (Leica DM IRB).

Western blot analysis. Total protein was isolated from cells from each group by using RIPA lysis buffer and PMSF at a ratio of 100:1 and the protein concentration was detected by BCA protein assay kit (Beyotime Biotechnology). A total of $40 \mu \mathrm{g}$ of protein lysate for each sample was separated by using sodium dodecyl-sulfate polyacrylamide gel electrophoresis (SDS-PAGE), followed by electrophoretic transfer to PVDF membranes (Millipore, Billerica, MA, USA) at a constant current of $250 \mathrm{~mA}$ and the membranes were blocked with $5 \%$ nonfat milk for 1-2 $\mathrm{h}$. Then membranes were incubated with the first antibody at $4^{\circ} \mathrm{C}$ overnight, followed with HRP-conjugated goat anti-rabbit/mouse IgG (Cell Signaling Technology, Danvers, MA, USA). The proteins of interest were detected 
using BeyoECL plus reagent (Beyotime Biotechnology). The antibodies for western blot analyses were as follows: rabbit anti-DACH1 (Proteintech, Wuhan, China), mouse anti- $\beta$ actin, rabbit anti-Bcl-2, anti-caspase-3 and anti-PARP (Cell Signaling Technology), rabbit anti-E-cadherin, mouse anti$\mathrm{N}$-cadherin and rabbit anti-Vimentin (Abcam, Cambridge, MA, USA). The band intensity values were analyzed with Quantity One software. Results were recorded as fold changes compared to an internal reference standard.

Cell proliferation assay. To assess the influence of the interference plasmid transfections on cell growth rates, Capan-1 cells were seeded onto 96-well plates in triplicate at a density of 5000 cells/well and cultured overnight. Cells were transfected with three groups of plasmids respectively: pshRNA1-DACH1, which identified the highest inhibitory rate, the negative control pshRNA-NC and the empty vector pGenesil-1. At 24, 48, $72 \mathrm{~h}$ incubation after treated, $10 \mu \mathrm{l}$ CCK 8 solution was added to each well and wells containing medium without cells were used as blank control. The plates were incubated for further 2 h. Cell viability was measured with the Cell Counting Kit8 (CCK8, Dojindo Molecular Technologies, Japan). The optical density (OD) values were measured at $450 \mathrm{~nm}$ and data are shown as means $\pm \mathrm{SD}$.

G418 concentration screening and colony formation assay. Capan-1 cells were cultured in a 24-well plate with a cell density of $1 \times 10^{4}$ each well. The screening concentrations of G418 were 400, 500, 600, 700, 800, 900, 1000, and $1100 \mu \mathrm{g} /$ $\mathrm{ml}$, and three replicates were made for each concentration. Then, a corresponding volume of G418 solution (Amresco, Solon, OH, USA) was added to each well combined with complement medium and cells, and changed with new culture medium every 2-3 days. After selection for two weeks, the minimum lethal concentration of G418 to Capan-1 cells was regarded as the final selection concentration.

Capan-1 cells were monolayer cultured in petri dishes until adherence confluence of $\sim 70 \%$, then transfected with pshRNA1-DACH1, pshRNA-NC and pGenesil-1 plasmid via Lipofectamine 2000. After $48 \mathrm{~h}$, cells with different treatments were separately collected, the suspension was inoculated into a new 6-well plate, which was selected for 2 weeks using G418 with a mini selection concentration of $800 \mu \mathrm{g} / \mathrm{ml}$. Culture medium and G418 were changed every 2-3 days. After 14 days of selection, cells were fixed with $4 \%$ paraformaldehyde for $30 \mathrm{~min}$, followed by $0.5 \%$ crystal violet staining for further $30 \mathrm{~min}$. Colonies with $>50$ cells were counted.

Detection of cell migration by Transwell assay. Cell mobility was evaluated by using a 24-well Transwell chamber with an $8 \mu \mathrm{m}$ pore-size PET membrane (Corning Inc., Corning, NY, USA). Briefly, cells were divided into three groups transfected with appropriate plasmids as mentioned above. After $48 \mathrm{~h}$ transfection, cells were digested with Trypsin/EDTA and collected from culture flasks. Capan-1 cells were then suspended in serum-free medium at a density of $2.5 \times 10^{5}$ cells $/ \mathrm{ml}$ and $200 \mu \mathrm{l}$ cell suspension was added into the upper well, and $700 \mu \mathrm{l}$ of the complete medium containing $10 \%$ FBS was placed into the lower chamber. The 24-well plate was cultured at $37^{\circ} \mathrm{C}$, in $5 \% \mathrm{CO}_{2}$ incubator for $24 \mathrm{~h}$. Cells that migrated from the upper chamber to the lower
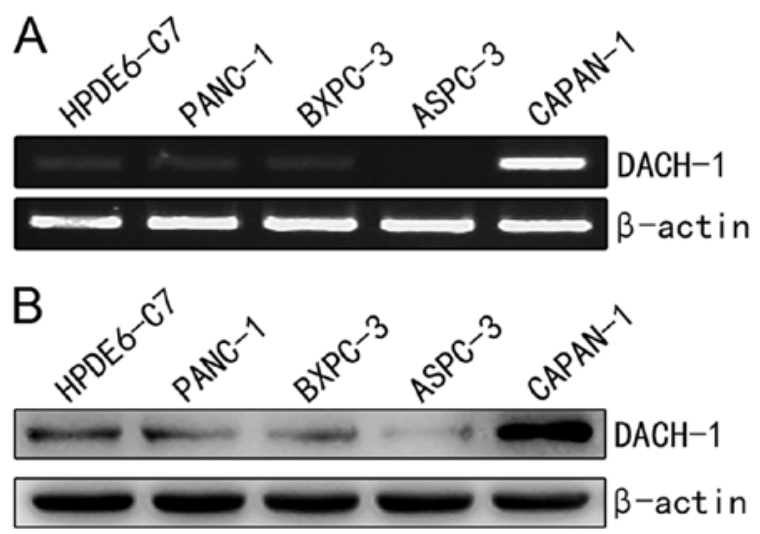

Figure 1. Expression of DACH1 in the normal epithelial HPDE6-c7 cells and pancreatic cancer cell lines (PANC-1, BxPC-3, AsPC-1 and Capan-1) were examined by RT-PCR (A) and western blotting (B), with $\beta$-actin as a control.

surface of the PET membrane were fixed with $40 \mathrm{~g} / 1$ paraformaldehyde for $30 \mathrm{~min}$ and then stained with $1 \mathrm{~g} / \mathrm{l}$ crystal violet for another $30 \mathrm{~min}$. Finally, the Transwell chamber was washed with PBS buffer and five random visual fields were counted to calculate the average number under a light microscope (x200).

Transwell invasion assay. To detect the effect of knockdown of DACH1 on cell invasion ability, 24-well Transwell chambers ( $8 \mu \mathrm{m}$ pore-size) were used. Matrigel (BD Biosciences, San Jose, CA, USA) was mixed with cold DMEM without fetal bovine serum at a ratio of 1:7, which simulates the extracellular matrix structure. Then the mixtures were loaded onto the upper chamber and incubated at $37^{\circ} \mathrm{C}$ for $4 \mathrm{~h}$. Capan-1 cells, transfected with the pshRNA-DACH1, pshRNA-NC and vector plasmids, were collected and suspended in serum-free medium. When the mixtures converted to solid state, approximately $3 \times 10^{4}$ cells from each group were planted into the upper compartment and allowed to penetrate to the lower well containing complete medium with $10 \%$ FBS. After incubation for $36 \mathrm{~h}$ at $37^{\circ} \mathrm{C}$ humidified atmosphere with $5 \% \mathrm{CO}_{2}$, cells on the upper side of the filter were wiped off with cotton swabs, subsequently, the invaded cells on the bottom of the PET membrane were fixed with paraformaldehyde then stained with crystal violet as above. The numbers of the invasion cell were counted at five different areas.

Cell cycle and apoptosis by flow cytometer. The pshRNADACH1, pshRNA-NC and vector plasmids were separately transfected into Capan-1 cells as described above. For cell cycle analysis, cells were treated for $48 \mathrm{~h}$, later digested by Trypsin without EDTA, suspended and washed twice by precold PBS buffer, then fixed in ice-cold $70 \%$ ethanol at $4^{\circ} \mathrm{C}$ overnight and analyzed by flow cytometer in Academy of Life Science, Chongqing Medical University. For assessing cell apoptosis, three groups of cells were cultured for $48 \mathrm{~h}$, digested with Trypsin/EDTA-free, suspended in PBS buffer and flow cytometry analysis was immediately performed. Each experiment was repeated three times.

Statistical analysis. Statistical analysis was performed using SPSS version 18.0 software. Measurement data were 
Table I. Sequences of the four shRNA targeting DACH1.

$\begin{array}{ll}\text { Target sequences } & \text { Nucleotide sequences }\end{array}$

1. ACTCTCACATCATGCCGCATT

5'-GATCCCGACTCTCACATCATGCCGCATTTT CGAATGCGGCATGATGTGAGAGTTTTTTA-3'

5'-AGCTTAAAAAACTCTCACATCATGCCGCAT TCGAAAATGCGGCATGATGTGAGAGTCGG-3'

2. CTGTTGAAAGTTGCCATAGAT

5'-GATCCCGCTGTTGAAAGTTGCCATAGATTT CGATCTATGGCAACTTTCAACAGTTTTTA-3'

5'-AGCTTAAAAACTGTTGAAAGTTGCCATAGA TCGAAATCTATGGCAACTTTCAACAGCGG-3'

3. GCACTTGAGTTTGAGACGAAA

5'-GATCCCGGCACTTGAGTTTGAGACGAAATT CGTTTCGTCTCAAACTCAAGTGCTTTTTA-3'

5'-AGCTTAAAAAGCACTTGAGTTTGAGACGAA ACGAATTTCGTCTCAAACTCAAGTGCCGG-3'

4. GAATCCGCACTACTCCTTACA

5'-GATCCCGGAATCCGCACTACTCCTTACATTC GTGTAAGGAGTAGTGCGGATTCTTTTTA-3'

(Negative control)

5'-AGCTTAAAAAGAATCCGCACTACTCCTTAC ACGAATGTAAGGAGTAGTGCGGATTCCGG-3'

presented as mean \pm SD. The expression difference of DACH1 between pancreatic cancer cell lines and normal cells was calculated by the Student's t-test, while comparison of three different treated groups was assessed using one-way analysis of variance. $\mathrm{P}<0.05$ was considered to indicate a statistically significant difference.

\section{Results}

DACH1 is overexpressed in Capan-1 cells compared to normal epithelial cells. DACH1 expression was altered in different cancers as previously described $(7,9,10,12)$. To explore the expression difference of DACH1 between pancreatic normal epithelial cell HPDE6-c7 cells and cancer cell lines (PANC-1, BxPC-3, AsPC-1, Capan-1), semi-quantitative RT-PCR and western blotting were performed. As shown in Fig. 1A, the mRNA level of DACH1 was significantly higher in Capan-1 cell line, compared with that in the immortalized epithelial cells. Moreover, DACH1 protein expression was further confirmed abundant in Capan-1 cancer cells (Fig. 1B), which correspond to the RT-PCR analysis. These results indicated that DACH1 was upregulated in Capan-1 pancreatic cancer cells, we assumed DACH1 might act as a cancer-promoting gene in pancreas, which may play an opposite role in tumor proliferation and in the growth of pancreatic cells as compared to cancer cells originating from other organs such as breast, prostate and stomach.

Identification of recombinant plasmid construction. Three DACH1-shRNA were designed and synthesized while a nonspecific shRNA as negative control (Table I), which were then inserted into pGenesil-1 vector plasmid. The recombinant plasmids and pGenesil-1 were identified by using EcoRI single enzyme digestion and EcoRI/HindIII double enzyme digestion.

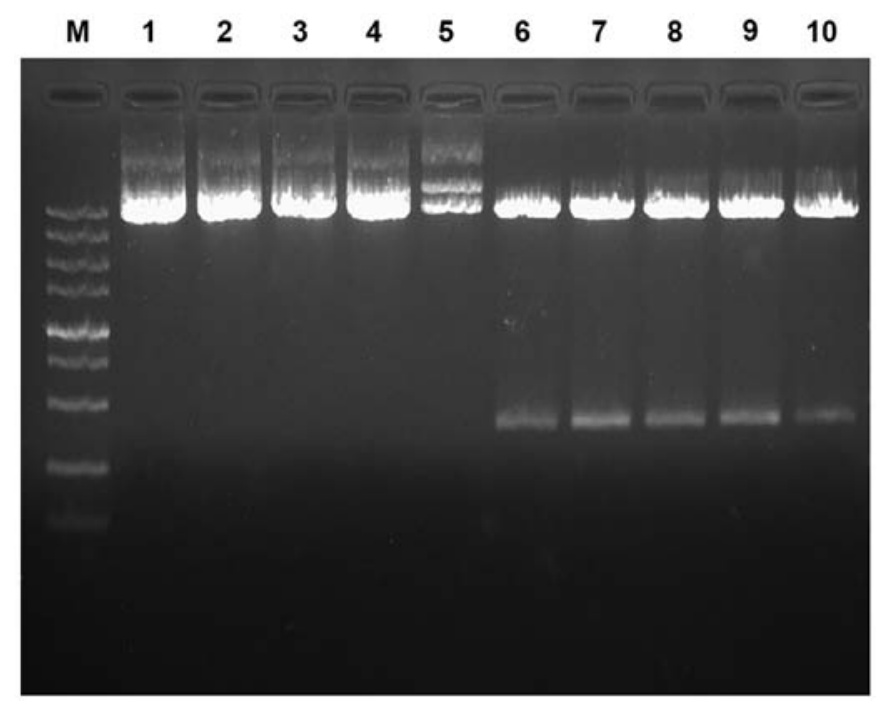

Figure 2. Identification of recombinant plasmid. Lane M: DNA marker (DL 10000); lanes 1-5: EcoRI single enzyme digestion of pshRNA1-DACH1, pshRNA2-DACH1, pshRNA3-DACH1, pshRNA-NC and pGenesil-1 plasmid; lanes 6-10: pshRNA1-DACH1, pshRNA2-DACH1, pshRNA3DACH1, pshRNA-negative control and pGenesil-1 plasmid were double digested by EcoRI and HindIII.

Our data demonstrated that with restriction endonuclease digestion, the DNA fragments of the four pshRNA-DACH1 were greater than pGenesil-1 (Fig. 2). Simultaneously, sequencing analysis showed that the inserted fragment completely matched with the expected sequence (Fig. 3). All these results confirmed that the interference plasmids were constructed successfully.

Knockdown efficiency of DACH1-shRNA1/2/3 sequences. To analyze the knockdown efficiency of the interference plasmid 

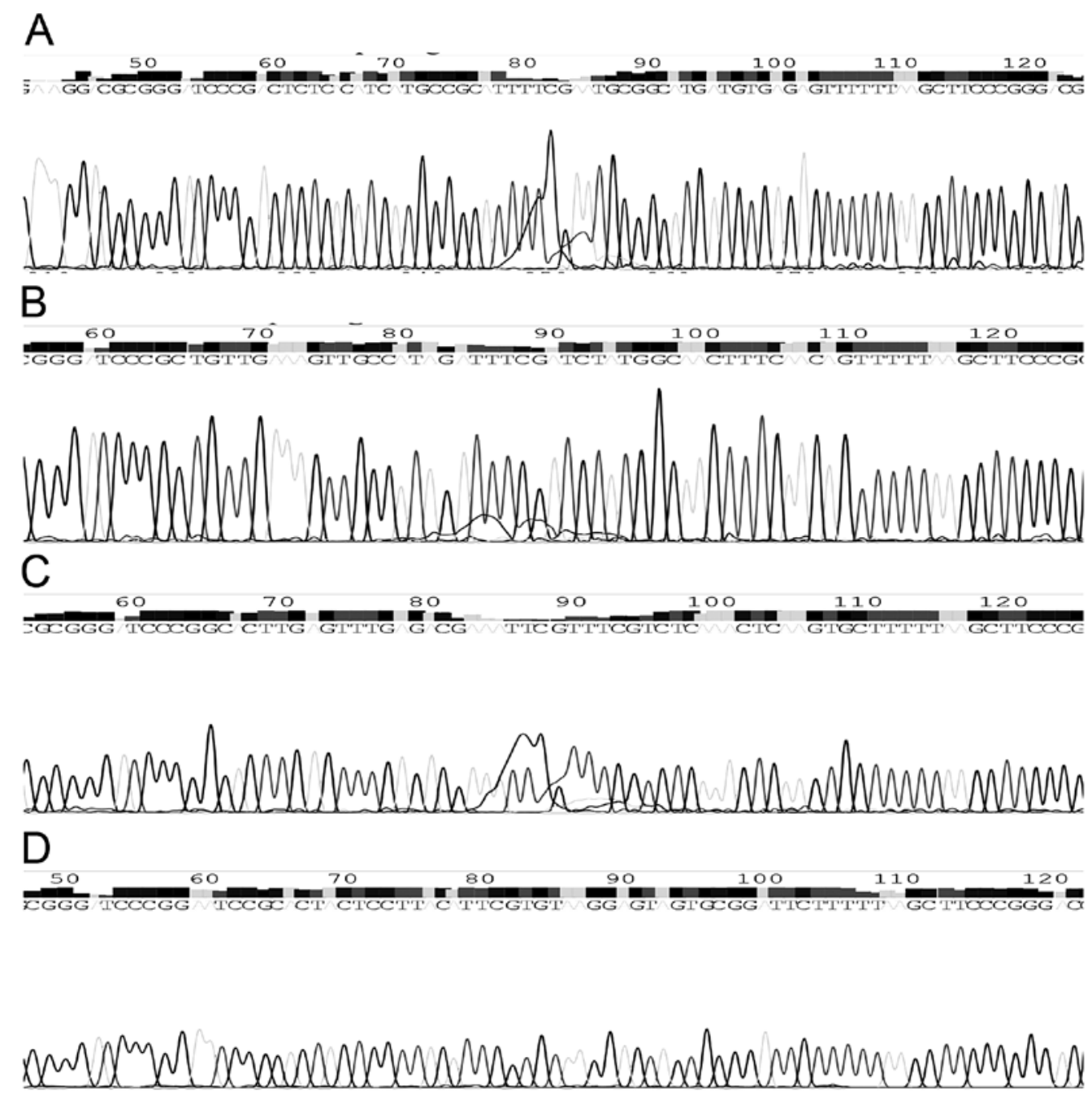

Figure 3. Sequencing graph. From A to D: shRNA1-DACH1, shRNA2-DACH1, shRNA3-DACH1, shRNA-NC plasmid.

targeting DACH1, samples were divided into six groups with different treatments: three interference groups (pshRNA1/2/3DACH1), pshRNA-NC, the empty plasmid pGenesil-1 and the untreated cell group. After $48 \mathrm{~h}$ transfection, the mRNA and protein levels of treated and untreated groups were, respectively, detected by RT-PCR and western blotting. As shown in Fig. 4A, the RNA expression of the six groups was lowest in the pshRNA1-DACH1, which was consistent with the protein level (Fig. 4B). Based on these statistics, we chose pshRNA1DACH1 as the most efficient interference plasmid to complete the following experiment.

RNAi-mediated knockdown of DACHI inhibits the proliferation and growth of Capan-1 pancreatic cancer cells. The effect of shRNA targeting DACH1 on cancer cell proliferation and growth was then evaluated by using CCK8 assay and colony formation test. As shown in Fig. 5, the survival colony number of the interference group compared to the negative control was $56 \pm 14$ versus $144 \pm 24(\mathrm{P}<0.01)$, indicating $\sim 60 \%$ reduction in RNAi-mediated transfected cells. To further confirm the cell viability, CCK-8 kit was used in accordance with the instruction steps. The OD values were measured with enzyme-link meters at $450 \mathrm{~nm}$ and recorded in Table II.
Table II. Effect of recombinant plasmids on cell proliferation. ${ }^{\mathrm{a}}$

\begin{tabular}{lccc}
\hline Plasmids & $24 \mathrm{~h}$ & $48 \mathrm{~h}$ & $72 \mathrm{~h}$ \\
\hline pshRNA-DACH1 & $0.49 \pm 0.013$ & $0.51 \pm 0.020$ & $0.60 \pm 0.029$ \\
pshRNA-NC & $0.61 \pm 0.020$ & $0.89 \pm 0.002$ & $1.16 \pm 0.027$ \\
pGenesil-1 & $0.63 \pm 0.029$ & $0.87 \pm 0.029$ & $1.08 \pm 0.094$
\end{tabular}

${ }^{a}$ Means $\pm \mathrm{SD}, \mathrm{n}=3$.

The statistics showed that cell proliferation rates were all significantly reduced after $24 \mathrm{~h}, 48$ or $72 \mathrm{~h}$ transfection with pshRNA1-DACH1 in Capan-1 cells (Fig. 6). Therefore, the results demonstrated that knockdown of DACH1 could inhibit the proliferation and growth of Capan-1 cells.

Effect of downregulating DACH1 expression on Capan-1 cancer cell cycle, apoptosis and the apoptotic markers. Cell cycle and apoptosis were analyzed by flow cytometer with Annexin V-FITC/PI staining. Results of cell cycle distribution among pshRNA-, control- and vector-transfected groups have 


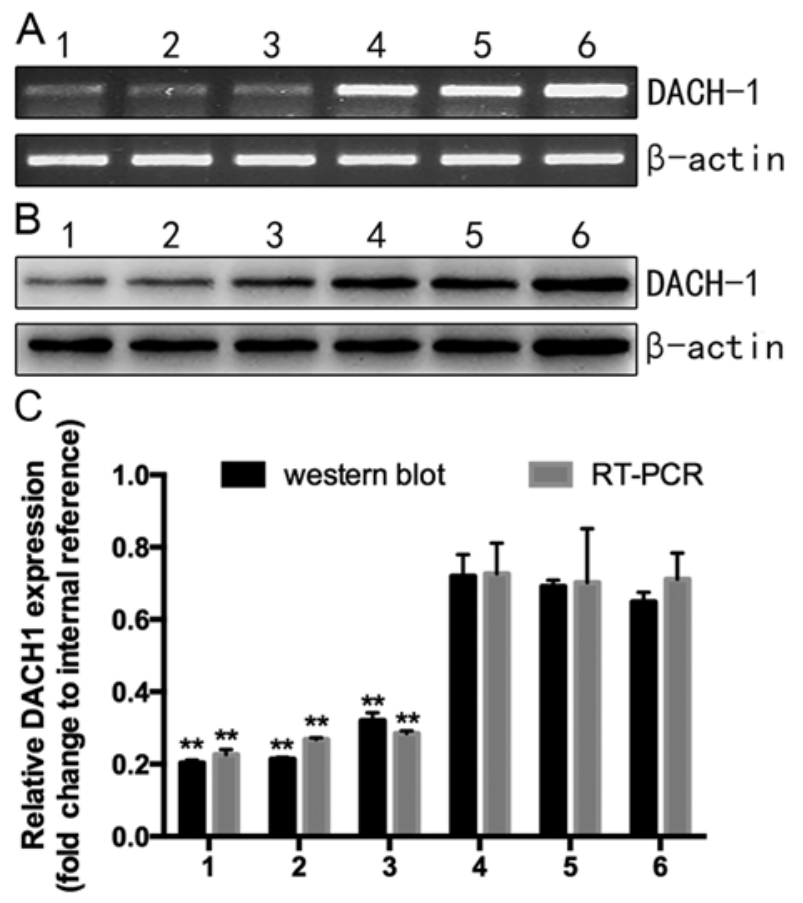

Figure 4. Analysis of the knockdown efficiency of the shRNA targeting DACH1 expression in Capan-1 cells. (A) RT-PCR analysis of DACH1 expression. (B) DACH1 expression detected by western blotting. (C) Quantitative analysis of DACH1 expression in each group. Data are recorded as fold change to internal reference, and expressed as mean $\pm \mathrm{SD},{ }^{* *} \mathrm{P}<0.01$. The cells were all divided into six groups: lanes 1, pshRNA1-DACH1; 2, pshRNA2-DACH1; 3, pshRNA3-DACH1; 4, negative control; 5, pGenesil-1; 6, untreated group.

no significant difference (data not shown). However, representative results of apoptosis in different treatment groups are shown in Fig. 7, and revealed a significant increase of apoptotic cells in the pshRNA-transfected group in comparison with the control one. To further explore the significance of DACH1 on cell apoptosis and the involved mechanisms, we investigated the expression of apoptotic markers using western blotting. Our data showed that downregulation of the DACH1 activity inhibited Bcl2 accumulation in Capan-1 cancer cells, inducing its downstream target genes the cleaved-caspase 3 and cleaved poly (ADP-ribose) polymerase PARP expression, compared to the control groups (Fig. 8A and B). Taken together, these results indicated that knockdown of DACH1 expression could inhibit tumor cell proliferation and growth, induced cell apoptosis, which may function as a tumor-promoting gene in pancreatic cancer.

DACH1 modulates Capan-1 tumor cell migration and invasion via EMT. To determine whether DACH1 could influence tumor cellular mobility, Transwell assay was performed. In Transwell migration or invasion experiments, the invaded cell numbers were significantly less in the pshRNA1-DACH1 interference cells than the control cells $(\mathrm{P}<0.05)$ (Fig. 9A and B). Our results demonstrated DACH1 indeed possess the ability to promote metastasis in Capan-1 pancreatic cancer cells.

Tumor cell metastasis is a complex process with multiple factors, which are the biological properties of malignant carcinomas. Epithelial-mesenchymal transition (EMT) is known as a dynamic cellular process and often occurs during

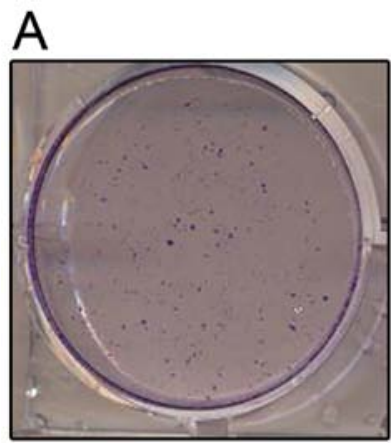

pshRNA-DACH1

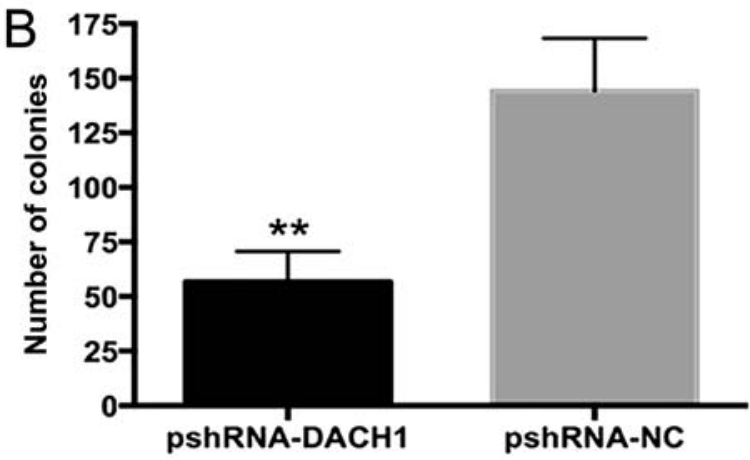

Figure 5. Colony formation assay. (A) The survival colony number of the shRNA-DACH1 cell group compared to the negative control group. (B) Quantitative analysis of the colonies in each group, ${ }^{* *} \mathrm{P}<0.01$.

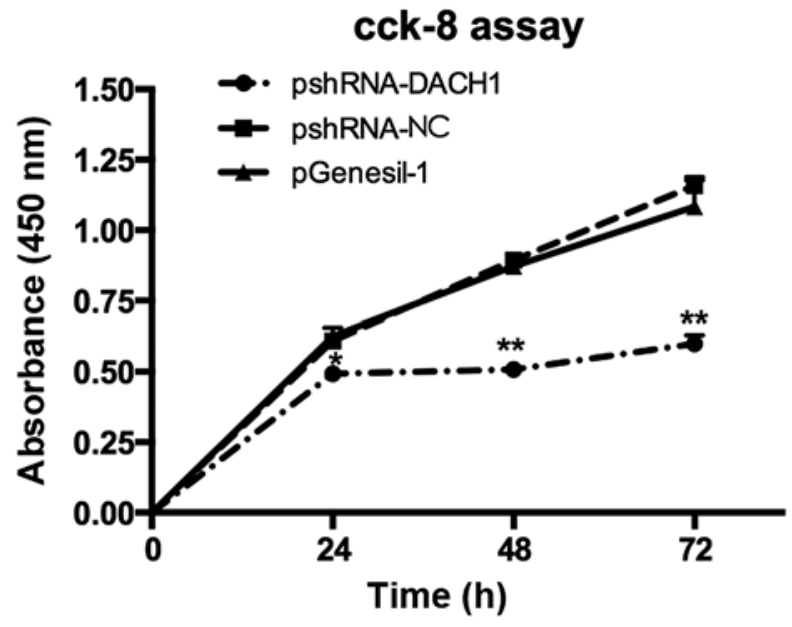

Figure 6. Capan-1 cell proliferation rate with pshRNA-DACH1, pshRNAnegative control and empty vector pGenesil-1 plasmids were analyzed by CCK-8 assay. Optical density was measured at $450 \mathrm{~nm},{ }^{*} \mathrm{P}<0.05,{ }^{* *} \mathrm{P}<0.01$.

cancer cell migration and invasion (13). To extend our analysis, we further examined whether downregulated expression of DACH1 in Capan-1 cancer cells was able to trigger a shift from mesenchyme to epithelia. As shown in Fig. 10A and $\mathrm{B}$, a remarkable increase of E-cadherin and reduction of $\mathrm{N}$-cadherin and Vimentin expression were detected in Capan-1 cells which were transfected with pshRNA-DACH1 plasmid. These findings suggested that knockdown the expression of DACH1 in Capan-1 cells could result in preventing EMT. 
pshRNA-DACH1
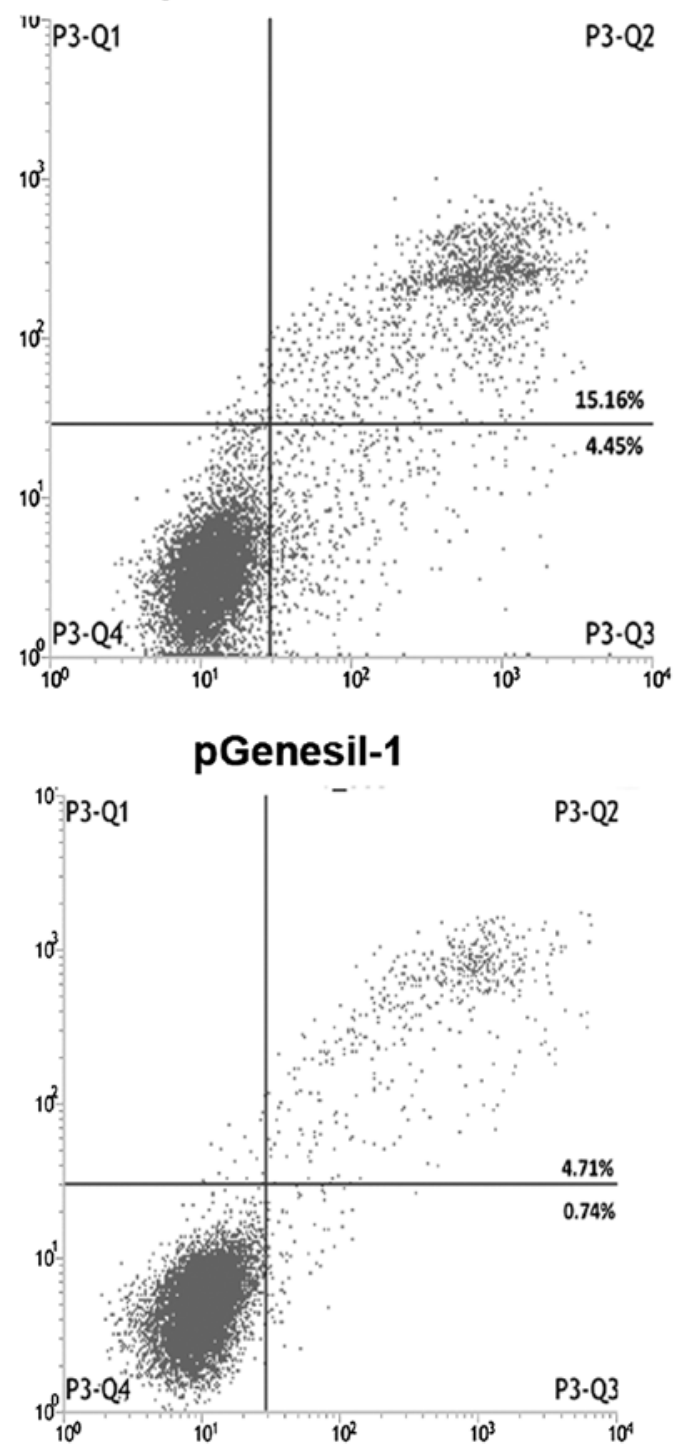

pshRNA-NC
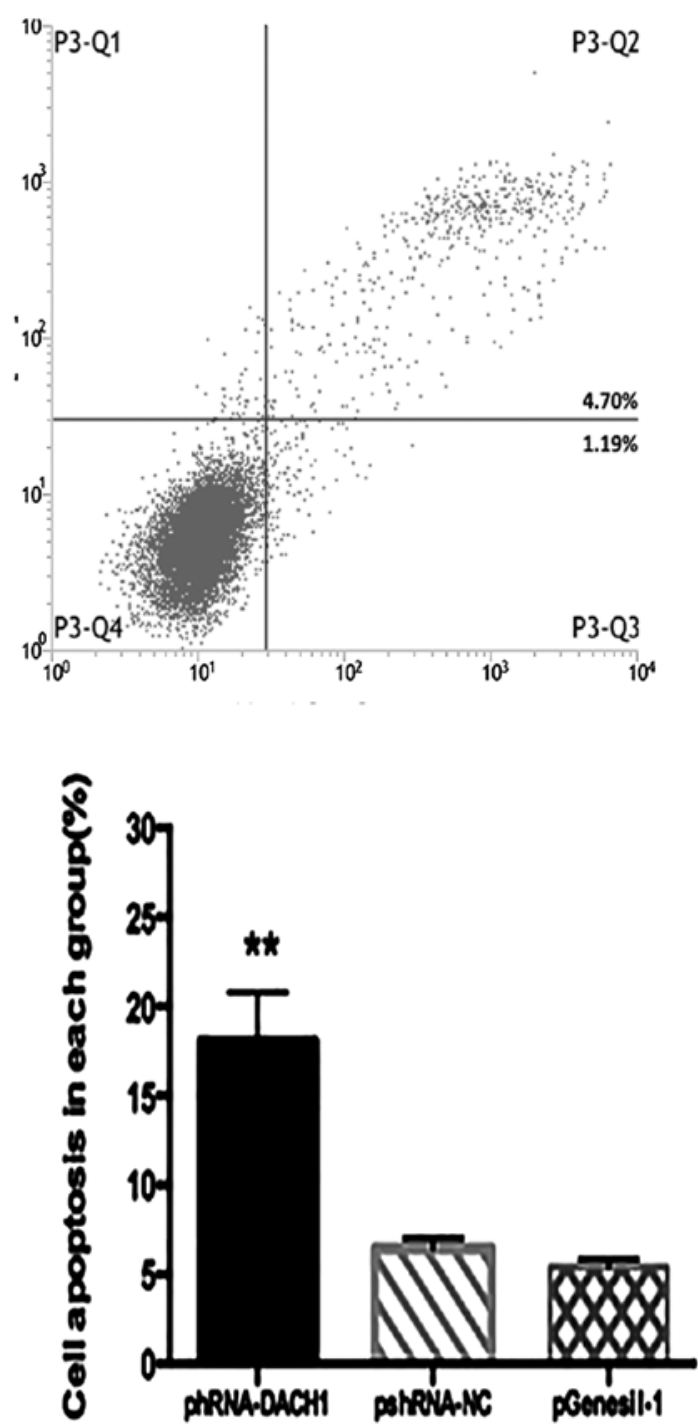

Figure 7. Analysis of the effect of DACH1 on cell apoptosis by flow cytometry. Cells were transfected with pshRNA-DACH1 plasmid, pshRNA-NC plasmid and pGenesil-1 plasmid. Summary of the flow cytometry data of cell apoptosis (last panel). ${ }^{* *} \mathrm{P}<0.01$.

\section{Discussion}

The incidence of pancreatic cancer has increased in recent years, ranking the sixth in China, which would bring us a huge burden of cancer-related death in the future $(14,15)$. To raise the survival rate and improve the therapeutic outcome, a multidisciplinary approach must be used to more effectively manage patients (3), including surgery, novel drug combinations, and radiation therapy. Fortunately, tremendous upswing in our understanding of the fundamental genetics provide hope to advance targeted therapy for the neoplasms of the pancreas. Currently, carbohydrate antigen (CA) 19-9 is the only predictive biomarker recognized in general for pancreatic cancer prognosis. However, in practical clinical application, it shows poor to moderate sensitivity and its specificity is limited to screenings of early pancreatic cancer (16).

The RDGN has been explored to integrate multiple signaling pathways, and is pivotal for the development of many organs such as eyes, muscle, ear, gonads and the central nervous system (17). It comprises several genes to govern tissue specification fate, mutation in any of these may contribute to the failure of normal development. The dachshund gene, a component of the RDGN, encodes conserved, nuclear proteins which play a prominent role in controlling retinal cell fate determination and leg development in Drosophila (18). The Dach/DACH, the mammalian homologues of the dachshund gene, was respectively isolated from mouse and human genes. It shared two highly conserved domains with Drosophila-Dachbox-N and Dachbox-C (19), which are likely to be functional domains. Dachbox-N is conserved with the N-terminal of Ski/Sno proto-oncogenes, which is known as DACH Ski/Sno (DS) domain and mediates DNA binding and transcriptional activation. Further investigations have identified that the synergistic effect between Eya and Dach among mouse homologues was not through a direct interaction, but was mediated by the involvement of CREB binding protein (CBP) (20). CBP is a transcription mediator 

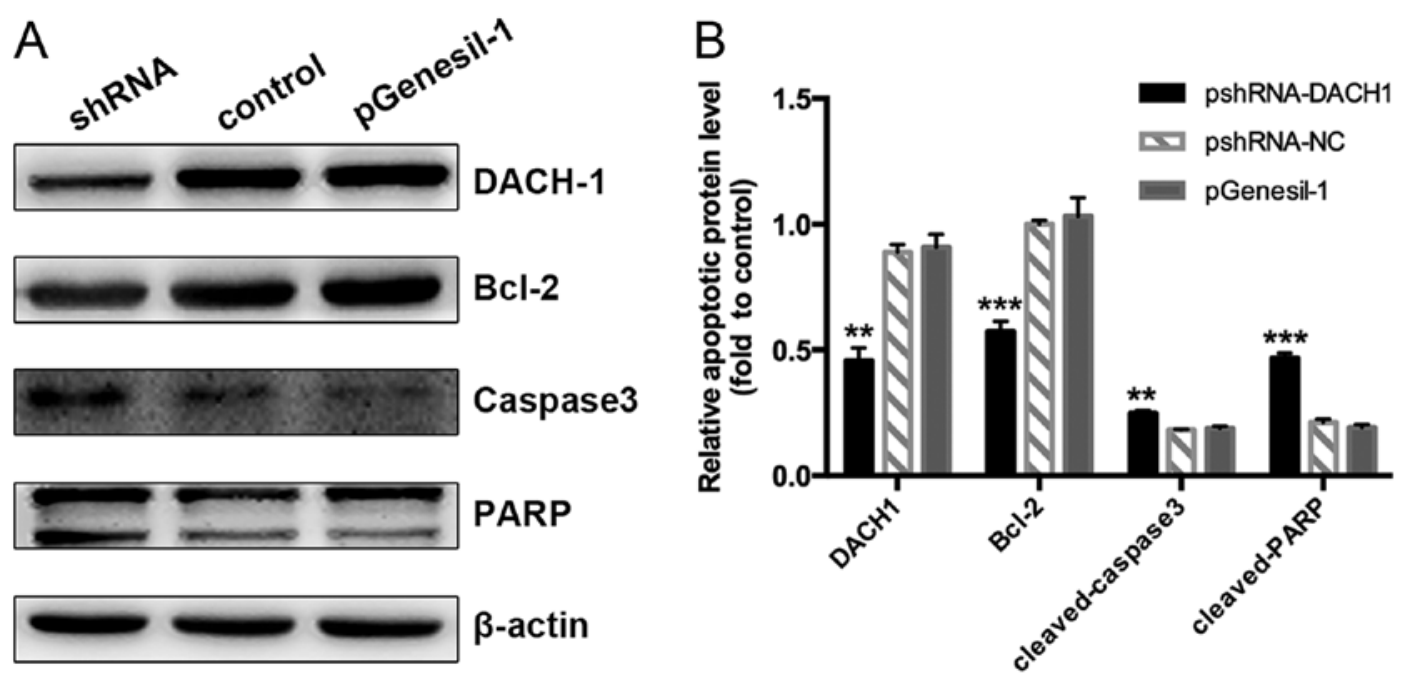

Figure 8. (A) Western blot analysis of apoptotic markers in pshRNA-, negative control-, and empty vector-transfected Capan-1 cells. $\beta$-actin was used as a control. (B) Quantitative analysis at molecular protein level in each group. Data are recorded as fold change to internal reference, ${ }^{* * *} \mathrm{P}<0.01,{ }^{* * *} \mathrm{P}<0.001$.

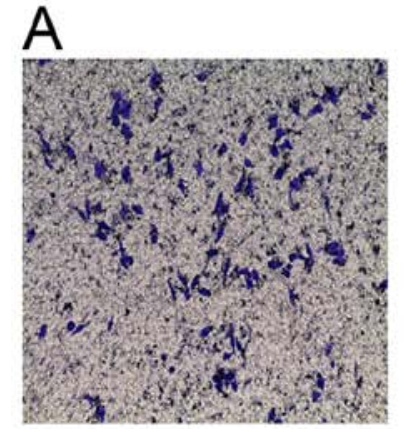

pShRNA-DACH1

B

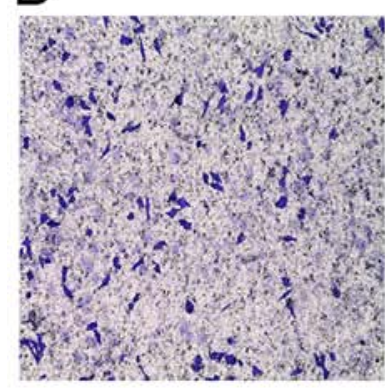

pshRNA-DACH1

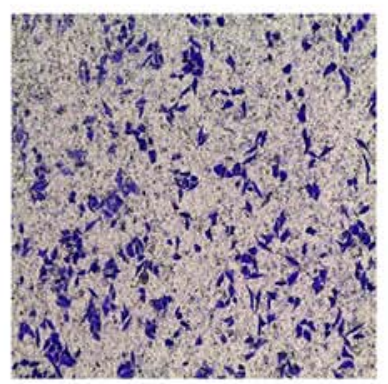

pshRNA-NC

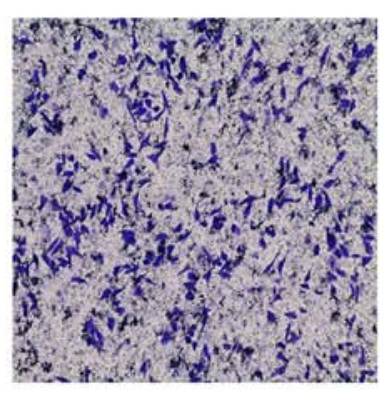

pshRNA-NC

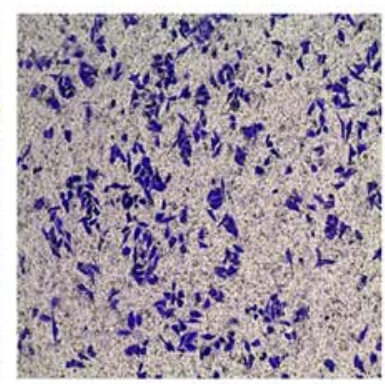

pGenesil-1

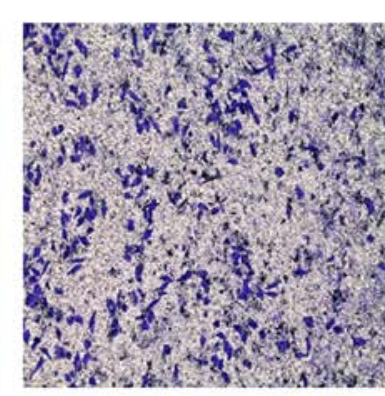

pGenesil-1
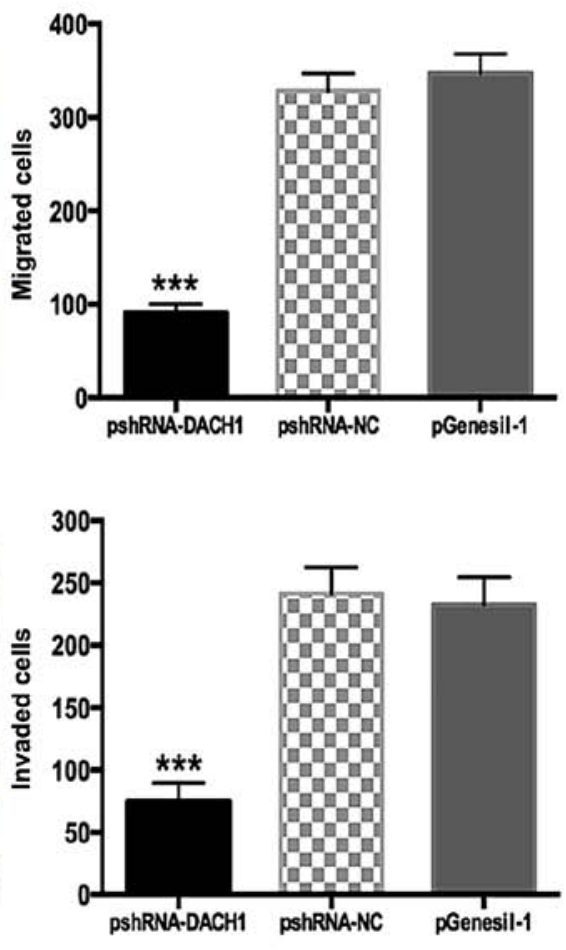

Figure 9. DACH1 regulates Capan-1 cell migration and invasion. (A) Transwell analysis of the effect of DACH1 on the Capan-1 cell migration after transfected with shRNA. Quantitative analysis of cell number through the membrane (right panel). (B) Cell mobility was detected with Transwell invasion assay. Summary of the invasion cell number (right panel). ${ }^{* * *} \mathrm{P}<0.001$.

involved in histone acetyltransferase (HAT) activity. However, the molecular mapping studies also found that DACH1 was coprecipitated with histone deacetylase 3 (HDAC3) (21). This suggests that the altered expression and function of DACH1 in different cancers is associated with its complex nature, such as it could either interact with HAT or histone deacetylase requires clarification.

In the present study, we analyzed the differential expression of DACH1 among four cancer cell lines and the normal counterparts at both mRNA and protein levels. The expression of DACH1 was abundant in Capan-1 pancreatic cancer cell lines, but downregulated in normal duct epithelial cell lines. Then, we applied RNA interference technology to investigate its function and related mechanisms in pancreatic cancer. As mentioned above, three recombinant interference plasmids and the negative control one were constructed successfully and transfected into Capan-1 cancer cells. CCK8 assay and flow cytometry analysis demonstrated that downregulation of DACH1 activity with shRNA indeed inhibits pancreatic cancer cell proliferation by mostly inducing apoptosis, but had 

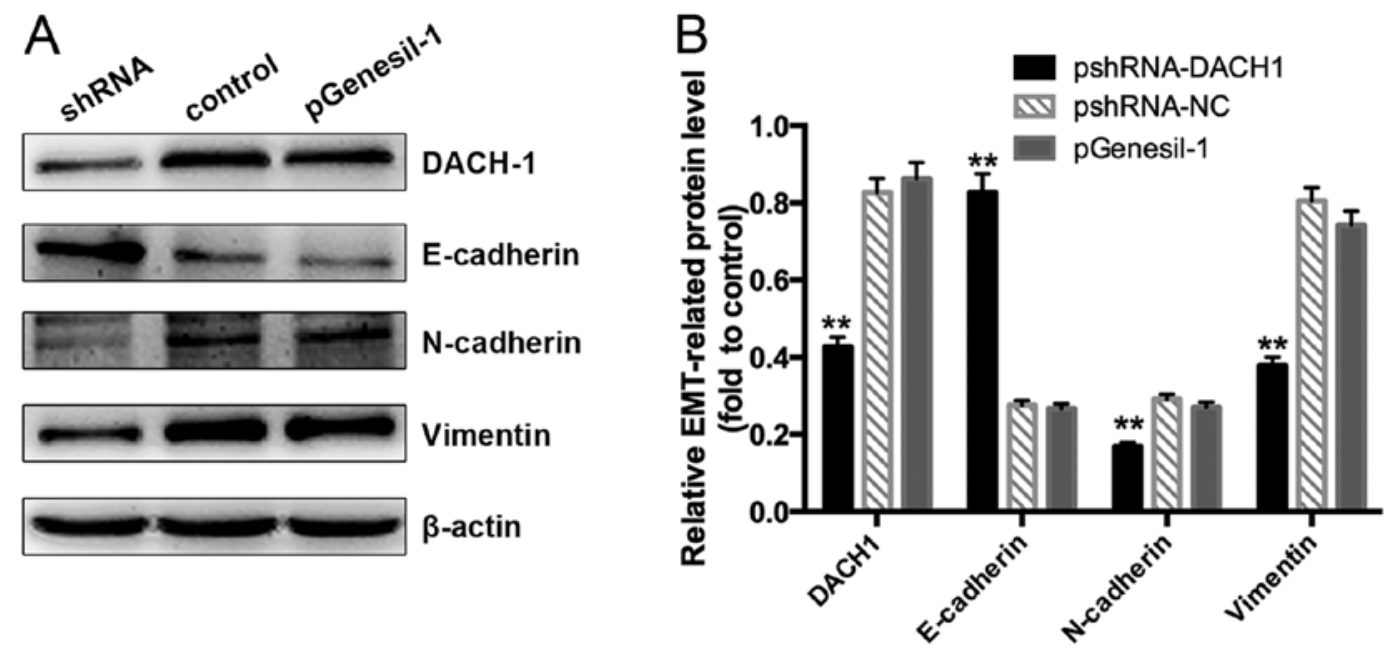

Figure 10. (A) EMT-related protein expression in pshRNA-, negative control-, and empty vector-transfected Capan-1 cells were detected with western blotting. (B) Quantitative analysis of EMT-related protein level with Fusion software. Data are recorded as fold change to internal reference, ${ }^{* *} \mathrm{P}<0.01$.

no effect on cell cycling. Apoptosis is a programmed cellular suicide mechanism and includes series of signal transduction molecules. Defects in the control of apoptosis are considered to be hallmarks of tumor genesis (22). The first discovery of a cell death regulator was cloned from human B-cell lymphomas, namely bcl-2 gene, which acts as a pro-survival regulator (23). Previous research has declared that deregulated expression of Bcl-2 and its related proteins may protect cells from apoptotic stimuli, leading to caspase activation, and subsequent tumor development and maintenance (24). Our data have shown that the decrease of $\mathrm{Bcl}-2$ protein was consistent with downregulation of DACH1, while the downstream genes cleaved-caspase 3 and PARP expression were upregulated. These findings raise a possibility that DACH1-mediated regulation of pancreatic cancer cell apoptosis activates Bcl-2 signaling axis and results in caspase cascade activation.

Additionally, we reported that knockdown of DACH1 suppressed Capan-1 cell migration and invasion through inhibiting the EMT process. E-cadherin is recognized as a characteristic molecule of the epithelial phenotype, which modulates the adherent junctions to promote desmosome formation and ultimately regulates cell morphogenesis (25). Loss of E-cadherin expression is observed in many poorly differentiated carcinomas, and parallels the expression of mesenchymal cell marker $\mathrm{N}$-cadherin, further indicating that EMT is correlated with the progression of primary tumors to invasive carcinoma $(26,27)$. In the past decades, studies have found that a zinc-finger protein belonging to Snail family controlled EMT and Snaill acted as a transcriptional repressor of the E-cadherin via binding to the E-box $(28,29)$. Recent research demonstrated that DACH1 expression suppressed EMT in breast cancer by modulating Snai1 (30). The regulation of epithelial or mesechymal markers is vital in EMT process and DACH1 inhibits carcinoma metastasis by interacting with the EMT factor Snail, thus, we explored whether DACH1 expression could regulate migration and EMT in pancreatic cancer. Corresponding to our speculation, our results demonstrated that knockdown of DACH1 activity repressed Capan-1 pancreatic cancer cell migration and invasiveness in Tranwell assay, furthermore, downregulation of DACH1 resulted in increased expression of the epithelial marker E-cadherin, simultaneously upregulation the two mesenchymal markers $\mathrm{N}$-cadherin and Vimentin. All these results supported our hypothesis that DACH1 expression plays an essential role in regulating cell invasive ability via EMT in pancreatic cancer cells. The detailed mechanism of the molecular interaction with DACH1 in regulating EMT is worthy of further investigation.

Collectively, our study indicates that DACH1 expression was upregulated in Capan-1 pancreatic cancer cells, and inhibition of its activity could remarkably repress cell proliferation and invasion. This suggests that DACH1 may function either as an oncogene or tumor suppressor gene in different tumor types. However, whether there are other molecular mechanisms and signaling pathways interacting with DACH1 in tumorigenesis in vivo or in vitro should be verified in future research.

\section{References}

1. Jemal A, Bray F, Center MM, Ferlay J, Ward E and Forman D: Global cancer statistics. CA Cancer J Clin 61: 69-90, 2011.

2. Zhang QH and Ni QX; Coordination Group of The Committee on Pancreatic Cancer: Clinical analysis of 2340 cases of pancreatic cancer. Zhonghua Yi Xue Za Zhi 84: 214-218, 2004 (In Chinese).

3. Wolfgang CL, Herman JM, Laheru DA, Klein AP, Erdek MA, Fishman EK and Hruban RH: Recent progress in pancreatic cancer. CA Cancer J Clin 63: 318-348, 2013.

4. Conroy T, Desseigne F, Ychou M, Bouché O, Guimbaud R, Bécouarn Y, Adenis A, Raoul JL, Gourgou-Bourgade S, de la Fouchardière $\mathrm{C}$, et al; Groupe Tumeurs Digestives of Unicancer; PRODIGE Intergroup: FOLFIRINOX versus gemcitabine for metastatic pancreatic cancer. N Engl J Med 364: 1817-1825, 2011.

5. Von Hoff DD, Ervin T, Arena FP, Chiorean EG, Infante J, Moore M, Seay T, Tjulandin SA, Ma WW, Saleh MN, et al: Increased survival in pancreatic cancer with nab-paclitaxel plus gemcitabine. N Engl J Med 369: 1691-1703, 2013.

6. Martik ML and McClay DR: Deployment of a retinal determination gene network drives directed cell migration in the sea urchin embryo. eLife 4: 4, 2015.

7. Wu K, Li A, Rao M, Liu M, Dailey V, Yang Y, Di Vizio D, Wang C, Lisanti MP, Sauter G, et al: DACH1 is a cell fate determination factor that inhibits cyclin D1 and breast tumor growth. Mol Cell Biol 26: 7116-7129, 2006.

8. Wu K, Katiyar S, Witkiewicz A, Li A, McCue P, Song LN, Tian L, Jin M and Pestell RG: The cell fate determination factor dachshund inhibits androgen receptor signaling and prostate cancer cellular growth. Cancer Res 69: 3347-3355, 2009. 
9. Yan W, Wu K, Herman JG, Brock MV, Zhou Y, Lu Y, Zhang Z, Yang Y and Guo M: Epigenetic silencing of DACH1 induces the invasion and metastasis of gastric cancer by activating TGF- $\beta$ signalling. J Cell Mol Med 18: 2499-2511, 2014.

10. Sunde JS, Donninger H, Wu K, Johnson ME, Pestell RG, Rose GS, Mok SC, Brady J, Bonome T and Birrer MJ: Expression profiling identifies altered expression of genes that contribute to the inhibition of transforming growth factor-beta signaling in ovarian cancer. Cancer Res 66: 8404-8412, 2006.

11. Lee JW, Kim HS, Kim S, Hwang J, Kim YH, Lim GY, Sohn WJ, Yoon SR, Kim JY, Park TS, et al: DACH1 regulates cell cycle progression of myeloid cells through the control of cyclin D, Cdk 4/6 and p21Cip1. Biochem Biophys Res Commun 420: 91-95, 2012.

12. Nan F, Lü Q, Zhou J, Cheng L, Popov VM, Wei S, Kong B, Pestell RG, Lisanti MP, Jiang J, et al: Altered expression of DACH1 and cyclin D1 in endometrial cancer. Cancer Biol Ther 8: 1534-1539, 2009.

13. Thiery JP, Acloque H, Huang RY and Nieto MA: Epithelialmesenchymal transitions in development and disease. Cell 139: 871-890, 2009.

14. Jemal A, Siegel R, Ward E, Hao Y, Xu J, Murray T and Thun MJ: Cancer statistics, 2008. CA Cancer J Clin 58: 71-96, 2008.

15. Ferlay J, Soerjomataram I, Dikshit R, Eser S, Mathers C, Rebelo M, Parkin DM, Forman D and Bray F: Cancer incidence and mortality worldwide: Sources, methods and major patterns in GLOBOCAN 2012. Int J Cancer 136: E359-E386, 2015.

16. Chen Y, Hao J, Ma W, Tang Y, Gao C and Hao X: Improvement in treatment and outcome of pancreatic ductal adenocarcinoma in north China. J Gastrointest Surg 15: 1026-1034, 2011.

17. Popov VM, Wu K, Zhou J, Powell MJ, Mardon G, Wang C and Pestell RG: The Dachshund gene in development and hormoneresponsive tumorigenesis. Trends Endocrinol Metab 21: 41-49, 2010.

18. Mardon G, Solomon NM and Rubin GM: dachshund encodes a nuclear protein required for normal eye and leg development in Drosophila. Development 120: 3473-3486, 1994.

19. Hammond KL, Hanson IM, Brown AG, Lettice LA and Hill RE: Mammalian and Drosophila dachshund genes are related to the Ski proto-oncogene and are expressed in eye and limb. Mech Dev 74: 121-131, 1998.
20. Ikeda K, Watanabe Y, Ohto $\mathrm{H}$ and Kawakami K: Molecular interaction and synergistic activation of a promoter by Six, Eya, and Dach proteins mediated through CREB binding protein. Mol Cell Biol 22: 6759-6766, 2002.

21. Wu K, Yang Y, Wang C, Davoli MA, D'Amico M, Li A, Cveklova K, Kozmik Z, Lisanti MP, Russell RG, et al: DACH1 inhibits transforming growth factor-beta signaling through binding Smad4. J Biol Chem 278: 51673-51684, 2003.

22. Hanahan D and Weinberg RA: The hallmarks of cancer. Cell 100: 57-70, 2000.

23. Tsujimoto Y, Finger LR, Yunis J, Nowell PC and Croce CM: Cloning of the chromosome breakpoint of neoplastic B cells with the $t(14 ; 18)$ chromosome translocation. Science 226: 1097-1099, 1984.

24. Kelly PN and Strasser A: The role of Bcl-2 and its pro-survival relatives in tumourigenesis and cancer therapy. Cell Death Differ 18: 1414-1424, 2011.

25. Batlle E and Wilkinson DG: Molecular mechanisms of cell segregation and boundary formation in development and tumorigenesis. Cold Spring Harb Perspect Biol 4: a008227, 2012.

26. Thiery JP: Epithelial-mesenchymal transitions in tumour progression. Nat Rev Cancer 2: 442-454, 2002.

27. Moustakas A and Heldin $\mathrm{CH}$ : Signaling networks guiding epithelial-mesenchymal transitions during embryogenesis and cancer progression. Cancer Sci 98: 1512-1520, 2007.

28. Wu Y and Zhou BP: Snail: More than EMT. Cell Adhes Migr 4: 199-203, 2010

29. Batlle E, Sancho E, Francí C, Domínguez D, Monfar M, Baulida J and García De Herreros A: The transcription factor snail is a repressor of E-cadherin gene expression in epithelial tumour cells. Nat Cell Biol 2: 84-89, 2000.

30. Zhao F, Wang M, Li S, Bai X, Bi H, Liu Y, Ao X, Jia Z and Wu H: DACH1 inhibits SNAI1-mediated epithelial-mesenchymal transition and represses breast carcinoma metastasis. Oncogenesis 4: e143, 2015 